\title{
Neutrino masses through the seesaw mechanism in 3-3-1 models
}

\author{
J. C. Montero, ${ }^{*}$ C. A. de S. Pires, ${ }^{\dagger}$ and V. Pleitez \\ Instituto de Física Teórica, Universidade Estadual Paulista, Rua Pamplona, 145, 01405-900 São Paulo, SP, Brazil
}

(Received 19 December 2001; published 15 April 2002)

\begin{abstract}
Some years ago it was shown by Ma that in the context of the electroweak standard model there are, at the tree level, only three ways to generate small neutrino masses by the seesaw mechanism via one effective dimension-five operator. Here we extend this approach to 3-3-1 chiral models showing that in this case there are several dimension-five operators and we also consider their tree level realization.
\end{abstract}

DOI: 10.1103/PhysRevD.65.095001

PACS number(s): 12.60.-i, 14.60.Pq

\section{INTRODUCTION}

Although recent data on neutrino oscillation experiments [1-3] strongly suggest that neutrinos have a nonzero small mass the issue of explaining its tiny value is still an open question. Several years ago it was noted by Weinberg [4], and independently by Wilczek and Zee [5], that the neutrinos may acquire naturally small Majorana masses through dimension-five effective operators such as

$$
\overline{l_{i a L}^{c}} l_{j b L} \Phi_{k}^{(m)} \Phi_{l}^{(n)}\left(f_{a b m n} \epsilon_{i k} \epsilon_{j l}+f_{a b m n}^{\prime} \epsilon_{i j} \epsilon_{j l}\right),
$$

the couplings $f$ and $f^{\prime}$ being of the order of $\Lambda^{-1}$, where $\Lambda$ is a large effective mass related to new physics. When the neutral component of the scalar doublet $\Phi$ develops its vacuum expectation value (VEV) $\langle\phi\rangle$ it will produce the following mass matrix for the neutrinos:

$$
M_{\nu}=\frac{f\langle\phi\rangle^{2}}{\Lambda}
$$

and since $\langle\phi\rangle$ is of the order of $100 \mathrm{GeV}$, small Majorana neutrino masses are generated if $\Lambda>10^{13} \mathrm{GeV}$. The interesting point in Eq. (2) is that the lightness of the neutrino masses are generated via new physics at an energy scale $\Lambda$ $\gg G_{F}^{-1 / 2}$. This is the usual seesaw mechanism [6]. The realizations of that operator inside the standard model (SM) were already investigated by Ma in Ref. [7]. All the ways such an operator can be realized at the tree and one loop level were sketched there.

Here we rederive step by step such realizations and extend the analysis to 3-3-1 chiral models [8-10] in which, like in the SM, neutrinos are massless unless we add righthanded neutrinos or break the lepton number. In this vain, after SuperKamiokande results, several suitable modifications of the model were already proposed in order to generate the neutrino masses $[11,12]$.

In this work we will build, in the framework of the 3-3-1 models, an effective dimension-five operator that leads to

\footnotetext{
*Email address: montero@ift.unesp.br

†Email address: cpires@ift.unesp.br

\#Email address: vicente@ift.unesp.br
}

seesaw neutrino masses and then investigate how it can be realized at the tree and one loop levels. Although it was noted in the early references $[4,5]$ that it is necessary to renormalize these effective operators, we will not address this issue here [13].

The outline of this work is the following. In Sec. II we review the several ways to generate such sort of operators in the context of the standard model. In Sec. III we consider the generation of those operators in the 3-3-1 model. Our conclusion will appear in Sec. IV.

\section{SEESAW TREE LEVEL REALIZATION IN THE STANDARD MODEL}

In the context of the standard model with only the lepton doublets $\Psi_{a L}=\left(\nu_{a L}, l_{a L}\right)^{T}, a=e, \mu, \tau$, and a scalar doublet $\Phi=\left(\phi^{+}, \phi^{0}\right)^{T}$, there are only three possibilities for implementing naturally small neutrino masses with dimension-five effective operators [7]. On the other hand, if we add new leptons new possibilities arise. They are the following.

(I) $\Psi_{a L}$ and $\Phi$ form a fermion singlet, so that the effective interaction is

$$
\begin{aligned}
\mathcal{L}_{1 F}^{e f f} & =\frac{f_{a b}}{\Lambda}\left[\overline{\epsilon\left(\Psi^{c}\right)_{a R}} \Phi\right]\left[\overline{\epsilon\left(\Psi^{c}\right)_{b R}} \Phi\right]+\text { H.c. } \\
& \left.=\frac{f_{a b}}{\Lambda} \overline{\left(\left(\nu^{c}\right)_{a R}\right.} \phi^{0}-\overline{\left(l^{c}\right)_{a R}} \phi^{+}\right)\left(\nu_{b L} \phi^{0}-l_{b L} \phi^{+}\right)+\text {H.c. }
\end{aligned}
$$

$\epsilon$ denotes the antisymmetric $S U(2)$ tensor and we have suppressed $S U(2)$ indices. The tree level realization of this effective operator is achieved by the introduction of one or more neutral right-handed singlets, say $N_{b R}$, which produces the usual seesaw mechanism [6], and is linked to the standard left-handed neutrinos through the interaction

$$
f_{a b} \bar{\Psi}_{a L} \widetilde{\Phi} N_{b R}+\text { H.c. }
$$

with $\widetilde{\Phi}=i \tau^{2} \Phi^{*}$; this interaction and the bare mass term for $N_{R}, \overline{\left(N_{a R}\right)^{c}} M_{R a b} N_{b R}$, leads to the following DiracMajorana matrix 


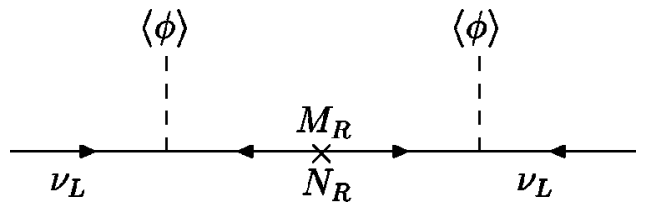

FIG. 1. Tree level realization of the effective dimension-five operator through a heavy neutrino.

$$
\left(\begin{array}{cc}
0 & M_{D}^{T} \\
M_{D} & M_{R}
\end{array}\right),
$$

in the basis $\left(\nu_{L},\left(N_{R}\right)^{c}\right)^{T}$. The matrix above, after diagonalization, gives the following expression for the neutrino masses:

$$
M_{\nu}=M_{D}^{T} M_{R}^{-1} M_{D} .
$$

From Eq. (6) we recover Eq. (2) by choosing $M_{D}$ $=f\langle\phi\rangle$ and taking $M_{R}=\Lambda$, obtaining in this way the canonical seesaw mechanism, or the now called type I [6], which is the usual realization of the effective operator in Eq. (1) found in the literature. Notice that the seesaw here is a relation among fermion masses and $N_{R}$ could be the right-handed neutrinos $\nu_{R}$. Its realization is depicted in Fig. 1 with the replacements $N_{R} \rightarrow \nu_{R}$ and $\langle\Phi\rangle \rightarrow\langle\phi\rangle$.

(II) $\Psi_{a L}$ and $\Psi_{b L}$ form a scalar triplet with the effective interaction

$$
\begin{aligned}
\mathcal{L}_{3 S}^{e f f}= & \frac{f_{a b}}{\Lambda}\left(\overline{\Psi^{c}}\right)_{a R} \Psi_{b L}(\epsilon \Phi \cdot \epsilon \Phi)+\text { H.c. } \\
= & \frac{f_{a b}}{\Lambda}\left[\overline{\left(\nu^{c}\right)_{a R}} \nu_{b L} \phi^{0} \phi^{0}-2 \phi^{0} \phi^{+}\left(\bar{\nu}_{a R}^{c} l_{b L}\right.\right. \\
& \left.\left.+\bar{l}_{a R}^{c} \nu_{b L}\right)+\bar{l}_{a R}^{c} l_{b L} \phi^{+} \phi^{+}\right]+ \text {H.c. }
\end{aligned}
$$

The tree level realization in this case is obtained by introducing a complex $(Y=2)$ scalar triplet $\left(\xi^{++}, \xi^{+}, \xi^{0}\right)$ or, in matrix notation $[14,15]$,

$$
\vec{\tau} \cdot \vec{\Xi}=\left(\begin{array}{cc}
\xi^{+} & \sqrt{2} \xi^{++} \\
\sqrt{2} \xi^{0} & -\xi^{+}
\end{array}\right) .
$$

In this case we have the interaction between the triplet $\Xi$ and the usual leptons,

$$
f_{a b} \bar{\Psi}_{a L}^{c}(\epsilon \vec{\tau} \cdot \Xi) \Psi_{b L}+\text { H.c. }
$$

From the expression above $\Xi$ must carry two units of lepton number. After the triplet develops a VEV the neutrinos gain masses which are given by the following expression:

$$
M_{\nu a b}=f_{a b}\left\langle\xi^{0}\right\rangle
$$

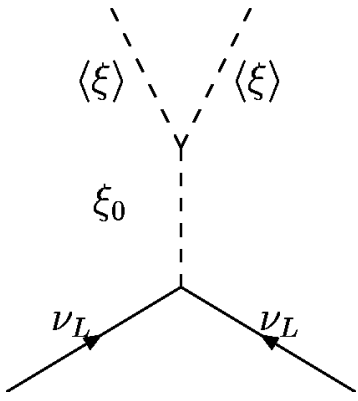

FIG. 2. Tree level realization of the effective dimension-five operator through a heavy scalar.

Differently from the type I seesaw mechanism here the suppression must come from $\left\langle\xi^{0}\right\rangle(\equiv\langle\xi\rangle)$. For this we have to study the scalar potential of the model. The complete scalar potential composed by the standard Higgs doublet $\Phi$ and the triplet $\Xi$ is

$$
\begin{aligned}
V(\phi, \xi)= & \mu_{\phi}^{2} \Phi^{\dagger} \Phi+\mu_{\Xi}^{2} \Xi^{\dagger} \Xi+\lambda_{\phi}\left(\Phi^{\dagger} \Phi\right)^{2} \\
& +\lambda_{\Xi}\left(\Xi^{\dagger} \Xi\right)^{2} \lambda_{\Xi \phi} \Xi^{\dagger} \Xi \Phi^{\dagger} \Phi+M_{\Xi} \Phi^{T} \Xi^{\dagger} \Phi,
\end{aligned}
$$

where the last term breaks explicitly the lepton number. The stationary conditions for this potential give the following constraint equations:

$$
\begin{array}{r}
\langle\phi\rangle\left(\mu_{\phi}^{2}+\lambda_{\phi}\langle\phi\rangle^{2}+\lambda_{\xi \phi}\langle\xi\rangle^{2}\right)=0, \\
\langle\xi\rangle\left(\mu_{\xi}^{2}+\lambda_{\xi}\langle\xi\rangle^{2}+\lambda_{\xi \phi}\langle\phi\rangle^{2}\right)+M_{\xi}\langle\phi\rangle^{2}=0 .
\end{array}
$$

Assuming that $\left|\mu_{\xi}\right| \sim M_{\xi} \gg\langle\phi\rangle$, with $\mu_{\xi}^{2}<0$, the second constraint equation provides the following relation between the vacua of the model:

$$
\langle\xi\rangle \sim \frac{\langle\phi\rangle^{2}}{M_{\xi}} .
$$

Substituting Eq. (13) in Eq. (10) we get the seesaw mass relation for neutrinos,

$$
M_{\nu a b}=\frac{f_{a b}\langle\phi\rangle^{2}}{M_{\xi}} .
$$

If the triplet $\xi$ belongs to some ground unified theory (GUT), we can recognize $M_{\xi}$ as the high scale $\Lambda$. This is the so-called type II seesaw mechanism [16]. Notice that in this type of seesaw a very heavy scalar could develop a very tiny vacuum $[7,17]$, differently of the type I seesaw where a very heavy right-handed neutrino induces tiny mass to the light neutrinos. The tree level realization of this mechanism is shown in Fig. 2.

(III) $\Psi_{a L}$ and $\Phi$ form a fermion triplet with $Y=0$ and the effective Lagrangian is then written as 


$$
\begin{aligned}
\mathcal{L}_{3 F}^{e f f}= & \frac{f_{a b}}{\Lambda}\left[\overline{\left(\Psi^{c}\right)_{a R}} \cdot \Phi\right]\left(\epsilon \Psi_{b L} \cdot \epsilon \Phi\right)+\text { H.c. } \\
= & \frac{f_{a b}}{\Lambda}\left[\bar{\nu}_{a R}^{c} l_{b L} \phi^{+} \phi^{0}-\left(\bar{\nu}_{a R}^{c} \phi^{0}+\bar{l}_{a R}^{c} \phi^{+}\right)\left(l_{b L} \phi^{+}\right.\right. \\
& \left.\left.+\nu_{b L} \phi^{0}\right)+\bar{l}_{a R}^{c} \nu_{b L} \phi^{+} \phi^{0}\right]+ \text { H.c.. }
\end{aligned}
$$

In this case, the tree level realization is based on the introduction of the $Y=0$ triplet of leptons [18]:

$$
\vec{\tau} \cdot \vec{\Delta}_{L}=\left(\begin{array}{cc}
N & \sqrt{2} P^{+} \\
\sqrt{2} P^{-} & -N
\end{array}\right)_{L} .
$$

This triplet is linked to the standard model content by the interaction

$$
f_{a}\left[\widetilde{\Phi}^{T} \overline{\vec{\tau} \cdot \vec{\Delta}_{L}}\left(\Psi_{a L}\right)^{c}+\Phi^{T} \overline{\left(\vec{\tau} \cdot \Delta_{L}\right)^{c}} \Psi_{a L}\right]+\text { H.c. }
$$

where $\widetilde{\Phi}=\epsilon \Phi^{*}$. The interaction in Eq. (17), together with the mass term $M_{\Delta} \overline{\left(\Delta_{L}\right)^{c}} \Delta_{L}$, yields the mass matrix, in the basis $\left(\nu_{a L}, N_{L}\right)^{T}$,

$$
\left(\begin{array}{cc}
0 & M_{L} \\
M_{L} & M_{\Delta}
\end{array}\right)
$$

Notice that in this case $M_{a L}=f_{a}\langle\phi\rangle$ is not an arbitrary mass matrix, hence, after diagonalization, two neutrinos are massless and two have nonzero masses at the tree level. One of the massive neutrinos has a mass proportional to $M_{\Delta}$ and the other one $\sim f_{a}\langle\phi\rangle^{2} / M_{\Delta}$ [18]. Although this is also a seesaw relation we see that the neutrino mass spectrum is different from that of the general seesaw mechanism, since two of them are massless at the tree level. Notice also that the interactions in Eq. (17) violate explicitly the lepton number. This is the third possibility of realization at the tree level of the effective dimension-five operator that leads to a seesaw relation for the neutrino masses. Even though it leads to the same seesaw relation, as the last two most explored mechanisms explicited in (I) and (II), this mechanism has not been widely appreciated in literature. It is depicted in Fig. 1 with $N_{R}$ replaced by $\left(N_{L}\right)^{c}$.

An interesting version of the model in which we obtain a general seesaw mass matrix is the one where we identify $N_{L}$ as being $\left(\nu^{c}\right)_{L}$ and add three triplets $\Delta_{a L}, a=e, \mu, \tau$; since in this case $M_{L}=f_{a b}\langle\phi\rangle$, we can have a neutrino mass matrix as in Eq. (6) in the canonical seesaw mechanism.

Unlike the other two types of seesaw mechanisms, in the present one we must verify if this fermion triplet $\Delta$ does not lead to any implication in the charged lepton masses. The general mass matrix in the charged lepton sector is rather complicated since the interactions in Eq. (17) together with the standard Yukawa interaction $\lambda \bar{\Psi}_{L} \Phi l_{R}$ lead to the following charged lepton matrix in the basis $\left(l_{L}, P_{L}\right)$ :

$$
\left(\bar{l}_{L} \bar{P}_{L}\right)\left(\begin{array}{cc}
M_{D} & M_{L} \\
0 & M_{\Delta}
\end{array}\right)\left(\begin{array}{c}
l_{R} \\
P_{R}
\end{array}\right)
$$

where $M_{D a b}=\lambda_{a b}\langle\phi\rangle$. With $M_{a L}=f_{a}\langle\phi\rangle$ (or $M_{L}=f_{a b}\langle\phi\rangle$ if we add three triplets) we see that the mass matrix in Eq. (19) can be only easily diagonalized if we assume that $M_{D}$ is diagonal. For more details see Ref. [18].

(IV) However, if we extend the particle content of the standard model, another possibility is that $\Psi_{a R}^{c}$ and $\Phi$ form a non-Hermitian fermionic triplet with $Y=2$. In this case, although it is not possible to build an effective operator of the form given in Eq. (3), we can introduce a fermionic nonHermitian $(Y=2)$ triplet $\Omega=\left(\omega^{++}, \omega^{+}, \omega^{0}\right)$ or

$$
\vec{\tau} \cdot \vec{\Omega}=\left(\begin{array}{cc}
\omega^{+} & \sqrt{2} \omega^{++} \\
\sqrt{2} \omega^{0} & -\omega^{+}
\end{array}\right)_{L},
$$

so that we have the interactions

$$
f_{a}\left[\Phi^{T} \overline{\vec{\tau} \cdot \vec{\Omega}_{L}}\left(\Psi_{a L}\right)^{c}+\widetilde{\Phi}^{T} \overline{\left(\vec{\tau} \cdot \Omega_{L}\right)^{c}} \Psi_{a L}\right]+\text { H.c. }
$$

which together with a mass term $M_{\Omega} \overline{\left(\Omega^{c}\right)_{R}} \Omega_{L}$ give a mass matrix of the form in Eq. (5) with $M_{R} \rightarrow M_{\Omega}$. Next, we introduce the right-handed doublets as $\psi_{a R}=\left(N, E^{-}\right)_{a R}$ $\sim(2,-1)$, and so it is possible to have

$$
\mathcal{L}_{4 F}^{e f f}=\frac{f_{a b}}{\Lambda}\left(\overline{\Psi_{a L}} \cdot \Phi\right)\left(\psi_{b R} \cdot \widetilde{\Phi}\right)+\text { H.c. }
$$

Notice that, even if the first three ways of realization of the effective dimension-five operator at the tree level are very different, in the end the neutrino masses receive the same expression in the form of the seesaw relation presented in Eq. (2). In the fourth way it is also possible to generate such a mass matrix but only at the tree level if we do not restrict ourselves to the usual leptonic representation content. The only context in which they can be distinguished is by their consequences at high energy once the type I seesaw favors $S O(10)$ and type II and III favor $S U(5)$ in $\mathbf{1 5}$ and $\mathbf{2 4}$ representation, respectively. In fact, the first one has been studied more in the literature and it was in this context that the seesaw mechanism was proposed [6]. The scalar triplet has also been largely considered in literature [14]. Both alternatives can be implemented more naturally in the context of the standard model or in some of its extensions since, for instance, the introduction of neutral singlets leaves the model more symmetric relatively to quarks and leptons, or because the scalar representation content is not constrained by the gauge invariance. This is not the case with the lepton triplet. However, recently it has been shown that in a 3-3-1 supersymmetric model [19] there is a fermionic non-Hermitian triplet under the 3-2-1 symmetry that is part of a sextet of Higgssinos transforming as $(6,0)$ under $S U(3)_{L} \otimes U(1)_{N}$. 


\section{THE EFFECTIVE DIMENSION-FIVE OPERATOR AND ITS REALIZATION IN 3-3-1 CHIRAL MODELS}

In this section we build the effective dimension-five operator in the context of 3-3-1 chiral models and its realizations at the tree and the 1-loop level. We employ the same approach used for the realizations of Eq. (2) in the SM outlined in the last section. As in the SM, in the minimal 3-3-1 model [8], or in some of its extensions [12], neutrinos are strictly massless due to the conservation of the total lepton number. In these models all leptons come only in triplets. In one version of this model, to generate the mass of all the particle content of the model, three triplets $\eta, \rho, \chi$ and a sextet $S$ of scalars are required.

We have at least two triplets, the leptonic one $\Psi_{a L}$ $=\left(\nu_{a L}, l_{a L}, l_{a L}^{c}\right)^{T}$ and the scalar one $\eta=\left(\eta^{0}, \eta_{1}^{-}, \eta_{2}^{+}\right)^{T}$, both transforming like $(\mathbf{3}, 0)$ under the electroweak gauge symmetry $S U(3)_{L} \otimes U(1)_{N}$. Hence, we can form bilinears like $\bar{\Psi} \eta=\overline{\mathbf{3}} \otimes \mathbf{3}=\mathbf{1}_{A} \oplus \mathbf{8}$ or $\Psi \eta=\mathbf{3} \otimes \mathbf{3}=\overline{\mathbf{3}}_{A} \oplus \mathbf{6}_{S}$. In this case we will have the following possibilities for the effective interactions:

(A) $\Psi$ and $\eta$ form a fermion singlet, and the effective operator is written as

$$
\begin{aligned}
\mathcal{L}_{1 F}^{e f f}= & \frac{f_{a b}}{\Lambda}\left[\eta^{\dagger} \overline{\left(\Psi^{c}\right)_{a R}}\right]\left[\eta^{\dagger} \Psi_{b L}\right]+\text { H.c. } \\
= & \frac{f_{a b}}{\Lambda}\left(\bar{\nu}_{a R}^{c} \eta^{0 *}+\bar{l}_{a R}^{c} \eta_{1}^{+}+\bar{l}_{a R} \eta_{2}^{-}\right)\left(\nu_{b L} \eta^{0 *}\right. \\
& \left.+l_{b L} \eta_{1}^{+}+l_{b L}^{c} \eta_{2}^{-}\right)+ \text {H.c. }
\end{aligned}
$$

At the tree level we can realize this situation by introducing again one or more neutral fermion singlets, $N_{b R}$ 's, and it is also a realization of the usual seesaw mechanism. This is in fact equivalent to case (I) in the previous section. Like there the intermediate heavy particle here should be a righthanded neutrino, $\nu_{R}$. The realization of this operator with $\nu_{R}$ is similar to that in Fig. 1 with $\langle\phi\rangle$ replaced by $\langle\eta\rangle$. The heavy right-handed neutrinos are linked with $\nu_{L}$ by a term like this one:

$$
f_{a b} \bar{\Psi}_{a L} \eta \nu_{b R}+\text { H.c. }
$$

which together with the mass term $M_{R} \bar{\nu}_{R}^{c} \nu_{R}$ leads to the mass matrix defined in Eq. (5) in the basis $\left(\nu_{L},\left(\nu_{R}\right)^{c}\right)$ with $M_{D a b}=f_{a b}\langle\eta\rangle$.

(B) Next, we can form with $\Psi_{a L}$ and $\eta$ an octet of leptons if we define the traceless matrix,

$$
\bar{M}_{a i}^{j}=\overline{\left(\Psi^{c}\right)_{a i R}} \eta^{\dagger j}-\frac{1}{3} \delta_{i}^{j}\left(\overline{\left(\Psi^{c}\right)_{a R}} \cdot \eta^{\dagger}\right)
$$

where $i, j$ denote $S U(3)$ indices. The effective interaction is in this case given by

$$
\begin{aligned}
\mathcal{L}_{8 F}^{e f f}= & \frac{f_{a b}}{\Lambda} \operatorname{Tr}\left(\bar{M}_{a} M_{b}\right)+\text { H.c. } \\
= & \frac{f_{a b}}{\Lambda}\left[\frac{1}{9}\left(2 \bar{\nu}_{q R}^{c} \eta^{0 *}-\bar{l}_{a R}^{c} \eta_{1}^{+}-\bar{l}_{a R} \eta_{2}^{-}\right)\left(2 \nu_{b L} \eta^{0}-l_{b L}^{c} \eta_{1}^{-}-l_{b L} \eta_{2}^{+}\right)+\bar{\nu}_{a R}^{c} \nu_{b:}\left(\eta_{1}^{+} \eta_{1}^{-}+\eta_{2}^{+} \eta_{2}^{-}\right)+\bar{l}_{a R}^{c} l_{b L}\right. \\
& \times\left(\eta^{0 *} \eta^{0}+\eta_{2}^{+} \eta_{2}^{-}\right)+\frac{1}{9}\left(2 \bar{l}_{a R}^{c} \eta_{1}^{+}-\bar{\nu}_{a R}^{c} \eta^{0 *}-\bar{l}_{a R} \eta_{2}^{-}\right)\left(2 l_{b L}^{c} \eta_{1}^{-}-\nu_{b L} \eta^{0}-l_{b L} \eta_{2}^{+}\right) \\
& \left.+\bar{l}_{a R}^{c} l_{b L}^{c} \eta_{2}^{-} \eta_{1}^{+}+\bar{l}_{a R} l_{b L}\left(\eta^{0 *} \eta^{0}+\eta_{1}^{+} \eta_{1}^{-}\right)+\frac{1}{9}\left(2 \bar{l}_{a R} \eta_{2}^{-}-\bar{l}_{a R}^{c} \eta_{1}^{+}-\bar{\nu}_{a R}^{c} \eta^{0 *}\right)\left(2 l_{b L} \eta_{2}^{+}-l_{b L}^{c} \eta_{1}^{-}-\nu_{b L} \eta^{0}\right)\right]+ \text { H.c. }
\end{aligned}
$$

Hence we need a heavy fermion octet transforming as $\mathbf{( 1 , 8 , 0 )}$ under the 3-3-1 factors,

$$
H_{L}=\left(\begin{array}{ccc}
N_{1} & P_{1}^{+} & P_{2}^{+} \\
P_{3}^{-} & N_{2} & X_{1}^{0} \\
P_{4}^{-} & X_{2}^{0} & N
\end{array}\right)_{L}
$$

where $N=-N_{1}-N_{2}$. We have the interaction

$$
f_{a}\left[\overline{\left(\Psi_{a L}\right)^{c}} H_{L} \eta+\eta^{\dagger} \overline{\left(H_{L}\right)^{c}} \Psi_{a L}\right]+\text { H.c. }
$$

omitting $S U(3)$ indices. There is also the bare mass term $M_{H} \overline{\left(H_{L}\right)^{c}} H_{L}$. These interactions produce a mass matrix for the neutral sector like that in Eq. (18), but now with $M_{a L}$ $=f_{a}\langle\eta\rangle$ and $M_{\Delta} \rightarrow M_{H}$. Two neutrinos remain massless and two gain masses. The tree level realization of this mechanism can be seen in Fig. 1 with $N_{R}$ replaced by $\left(N_{1,2 L}\right)^{c}$ and $\langle\phi\rangle$ replaced by $\langle\eta\rangle$. However, if we introduce three of such 
octets and identify, say $N_{1 a L}$ with $\left(\nu^{c}\right)_{a L}, a=e, \mu, \tau$, we can generate a general seesaw mass matrix like in Eq. (5) since now $M_{L a b}=f_{a b}\langle\eta\rangle$. Notice that although this case is the analog of case (III) in the previous section, i.e., an Hermitian, $Y=0$, fermion triplet, it is also a realization of case (IV) where there is a non-Hermitian, $Y=2$, fermion triplet under $S U(2)_{L} \otimes U(1)_{Y}$.

As in the case of the leptonic triplet in the standard model, the mass matrix of the charged lepton sector is complicated. Let us assume, for simplicity, that the mass matrix of the standard charged leptons, generated at low energy in the basis of the 3-3-1 symmetries, is diagonal and let us call it $M_{l}$, and for the mass matrix of the charged leptons, $P_{1}$ and $P_{2}$, matrix $M_{H}$. With these assumptions and the terms in Eq. (28), we have the following $5 \times 5$ matrix in the basis $\left(l_{L}, P_{1 L}, P_{2 L}\right)$ :

$$
\left(\begin{array}{cc}
M_{l} & M_{\eta} \\
0 & M_{H}
\end{array}\right) .
$$

As in case (III) in the previous section, the diagonalization of this matrix is complicated unless we assume that $M_{l}$ is diagonal; in this case the usual leptons decouple completely from the heavy ones, being the mass matrix of the former $M_{l}$ and that for the heaviest $M_{H}$.

(C) Finally, we have the possibility that $\Psi_{a R}^{c}$ and $\Psi_{b L}$ form a symmetric sextet. In this case we have

$$
\left.\mathcal{L}_{6 s}^{e f f}=\frac{f_{a b}}{\Lambda} \overline{\left(\left(\Psi^{c}\right)_{a R}\right.} \Psi_{b L} \eta^{\dagger} \eta^{\dagger}\right)+ \text { H.c. }
$$

and the tree level realization is the introduction of the usual sextet $(\mathbf{6}, 0)[8]$. Notice that the triplet $\epsilon_{i j k} \Psi_{j}^{c} \Psi_{k}$ is not real- ized since the respective triplet $\epsilon_{i l m} \eta_{l} \eta_{m}=0$. However, the symmetric sector can contribute for the masses of the charged leptons and also generate the neutrino masses by loop effects.

In this case the tree level realization requires a scalar sextet, $S$ :

$$
\left.S=\mid \begin{array}{lll}
s^{\prime} & \frac{s_{1}^{-}}{\sqrt{2}} & \frac{s_{2}^{+}}{\sqrt{2}} \\
\frac{s_{1}^{-}}{\sqrt{2}} & s_{3}^{--} & \frac{s}{\sqrt{2}} \\
\frac{s_{2}^{+}}{\sqrt{2}} & \frac{s}{\sqrt{2}} & s_{4}^{++}
\end{array}\right) \sim(\mathbf{1}, \mathbf{6}, 0) \text {. }
$$

The sextet $S$ is linked to the leptons by the interaction

$$
f_{a b} \bar{\Psi}_{a}^{c} S \Psi_{b}+\text { H.c. }
$$

When the neutral component $s^{\prime}$ of the sextet $S$ develops a $\mathrm{VEV}$, the neutrino mass matrix gets the following form:

$$
M_{\nu a b}=f_{a b}\left\langle s^{\prime}\right\rangle \text {. }
$$

The next step is to show how $\left\langle s^{\prime}\right\rangle$ gets a tiny value. For this we need to develop the scalar potential of the model taking into account the explicit terms that violate the lepton number. Let us first write the scalar potential that conserves the lepton number:

$$
\begin{aligned}
V(\eta, \rho, \chi, S)= & \mu_{\eta}^{2} \eta^{\dagger} \eta+\mu_{\rho}^{2} \rho^{\dagger} \rho+\mu_{\chi}^{2} \chi^{\dagger} \chi+\mu_{S}^{2} \operatorname{Tr}\left(S^{\dagger} S\right)+\lambda_{1}\left(\eta^{\dagger} \eta\right)^{2}+\lambda_{2}\left(\rho^{\dagger} \rho\right)^{2}+\lambda_{3}\left(\chi^{\dagger} \chi\right)^{2}+\left(\eta^{\dagger} \eta\right)\left(\lambda_{4}\left(\rho^{\dagger} \rho\right)+\lambda_{5}\left(\chi^{\dagger} \chi\right)\right) \\
& +\lambda_{6}\left(\rho^{\dagger} \rho\right)\left(\chi^{\dagger} \chi\right)+\lambda_{7}\left(\rho^{\dagger} \eta\right)\left(\eta^{\dagger} \rho\right)+\lambda_{8}\left(\chi^{\dagger} \eta\right)\left(\eta^{\dagger} \chi\right)+\lambda_{9}\left(\rho^{\dagger} \chi\right)\left(\chi^{\dagger} \rho\right)+\lambda_{10} \operatorname{Tr}\left(S^{\dagger} S\right)^{2}+\lambda_{11}\left(\operatorname{Tr}\left(S^{\dagger} S\right)\right)^{2} \\
& +\left(\lambda_{12}\left(\eta^{\dagger} \eta\right)+\lambda_{13}\left(\rho^{\dagger} \rho\right)\right) \operatorname{Tr}\left(S^{\dagger} S\right)+\lambda_{14}\left(\chi^{\dagger} \chi\right) \operatorname{Tr}\left(S^{\dagger} S\right)+\left(\lambda_{15} \epsilon^{i j k}\left(\chi^{\dagger} S\right)_{i} \chi_{j} \eta_{k}+\lambda_{16} \epsilon^{i j k}\left(\rho^{\dagger} S\right)_{i} \rho_{j} \eta_{k}\right. \\
& \left.+\lambda_{17} \epsilon^{i j k} \epsilon^{l m n} \eta_{n} \eta_{k} S_{l i} S_{m j}+\text { H.c. }\right)+\lambda_{18} \chi^{\dagger} S S^{\dagger} \chi+\lambda_{19} \eta^{\dagger} S S^{\dagger} \eta+\lambda_{20} \rho^{\dagger} S S^{\dagger} \rho .
\end{aligned}
$$

Here, for the sake of simplicity, we impose in the scalar potential the symmetry $\chi \rightarrow-\chi$ in order to avoid other trilinear terms besides the one that will generate the seesaw mechanism. There are also four terms permitted by the 3-3-1 gauge symmetry which violate explicitly the lepton number, but for what concerns us here we will just consider only one of them:

$$
M_{S} \eta^{T} S^{\dagger} \eta
$$

Adding this term to the potential in Eq. (34), we find the following stationary condition on the VEV of the scalar field $s^{\prime}$ :

$$
\begin{gathered}
\left\langle s^{\prime}\right\rangle\left[\mu_{S}^{2}+\lambda_{10}\langle s\rangle^{2}+\left(\frac{\lambda_{12}}{2}+\frac{\lambda_{19}}{2}\right)\langle\eta\rangle^{2}+\frac{\lambda_{13}}{2}\langle\rho\rangle^{2}+\frac{\lambda_{14}}{2}\langle\chi\rangle^{2}\right. \\
\left.+\left(\lambda_{10}+\frac{\lambda_{11}}{2}\right)\left\langle s^{\prime}\right\rangle^{2}\right]+M_{S}\langle\eta\rangle^{2}=0 .
\end{gathered}
$$

We will consider here the case when the charged leptons gain masses, not through a sextet but via the introduction of a heavy charged lepton [20]. In this case the sextet can be very heavy. For instance, we can assume $\mu_{S}^{2}<0$ and that $\left|\mu_{S}\right|=M_{S} \gg\langle\eta\rangle,\langle\rho\rangle,\langle\chi\rangle$, so that we have from Eq. (36) 


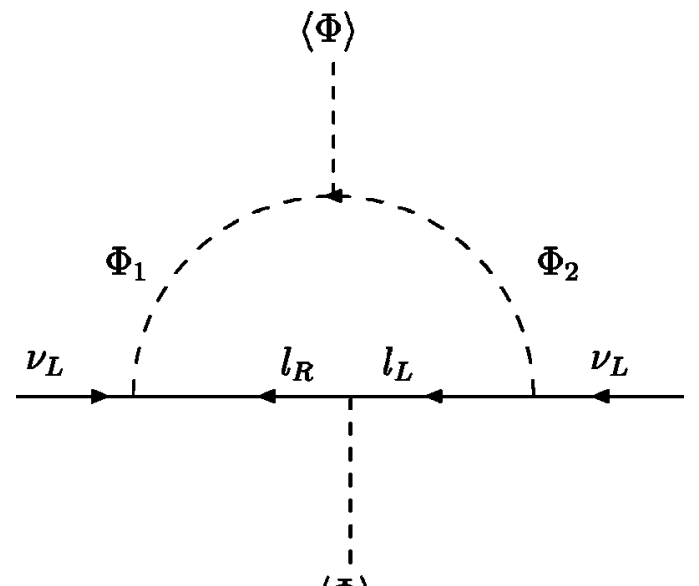

$\langle\Phi\rangle$

FIG. 3. One loop realization of the effective dimension-five operator.

$$
\left\langle s^{\prime}\right\rangle \simeq \frac{\langle\eta\rangle^{2}}{M_{S}},
$$

which gives the following expression to the neutrino mass matrix:

$$
M_{\nu a b}=f_{a b} \frac{\langle\eta\rangle^{2}}{M_{S}} .
$$

This is a seesaw mass relation and its realization is equal to that in Fig. 2 with $\langle\xi\rangle$ replaced by $\left\langle s^{\prime}\right\rangle$ and $\langle\phi\rangle$ replaced by $\langle\eta\rangle$. It is equivalent to the type II seesaw mechanism discussed in case (II) in the preceding section and in Ref. [17] where it was assumed that the sextet also gives mass to the charged leptons but the dominant energy scale is $\langle\chi\rangle$.

A sextet like that in Eq. (31) disposes of a second neutral component, $s$, which may contribute to the charged lepton masses when developing a VEV. However, it will not give a large contribution to the charged lepton masses as we can see in the following. From its stationary condition we get the constraint equation:

$$
\begin{aligned}
\langle s\rangle\left[\mu_{S}^{2}+\lambda_{10}\left\langle s^{\prime}\right\rangle^{2}+\left(\frac{\lambda_{12}}{2}-\lambda_{17}\right)\langle\eta\rangle^{2}+\left(\frac{\lambda_{13}}{2}+\frac{\lambda_{20}}{4}\right)\langle\rho\rangle^{2}\right. \\
\left.+\left(\frac{\lambda_{14}}{2}+\frac{\lambda_{18}}{4}\right)\langle\chi\rangle^{2}+\left(\frac{\lambda_{11}}{2}+\lambda_{10}\right)\langle s\rangle^{2}\right]+\frac{\lambda_{15}}{2 \sqrt{2}}\langle\eta\rangle\langle\chi\rangle^{2} \\
-\frac{\lambda_{16}}{2 \sqrt{2}}\langle\eta\rangle\langle\rho\rangle^{2}=0,
\end{aligned}
$$

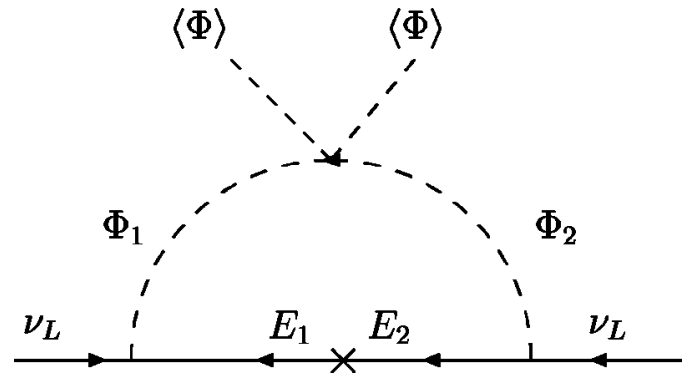

FIG. 4. Another one loop realization of the effective dimensionfive operator.

which gives the following expression to its vacuum:

$$
\langle s\rangle \simeq \frac{\langle\eta\rangle\langle\chi\rangle^{2}}{\mu_{S}^{2}} .
$$

It implies $\langle s\rangle \ll\left\langle s^{\prime}\right\rangle$ which makes its contribution to the charged lepton masses insignificant in relation to other sources in low energy. Notwithstanding, in the present case the charged leptons obtained mass through the mixing with a heavy charged lepton [9].

\section{CONCLUSIONS}

We have analyzed the realization at the tree level of an effective dimension-five operator that generates seesaw masses to the neutrinos and have added a new tree level implementation of the seesaw mechanism in the context of the standard electroweak model. From our analysis we can say that all the ways of realizing such an operator in the standard model can be easily implemented in the 3-3-1 model as well. In the particular case (IV) of Sec. II is realized in the context of the 3-3-1 model when a fermion octet is added as described in case (B) in Sec. III.

Finally, we would like to mention that at the one loop level the effective operator of the sort given in Eq. (1) is also easily implemented. Two simple cases are shown in Figs. 3 and 4, but they imply an extension of the 3-3-1 model by adding a pair of exotic lepton singlets. Only the second model is favored by phenomenology. Also we should stress that, except in case (B) the neutrinos and charged lepton masses share a common set of parameters which leave such scenarios interesting.

\section{ACKNOWLEDGMENTS}

This work was supported by Fundação de Amparo à Pesquisa do Estado de São Paulo (FAPESP), Conselho Nacional de Ciência e Tecnologia $(\mathrm{CNPq})$ and by Programa de Apoio a Núcleos de Excelência (PRONEX).
[1] Homestake Collaboration, B.T. Cleveland et al., Astrophys. J. 496, 505 (1998); Kamiokande Collaboration, Y. Fukuda et al., Phys. Rev. Lett. 77, 1683 (1996); GALLEX Collaboration, W. Hampel et al., Phys. Lett. B 477, 127 (1999); SAGE Collaboration, J.N. Abdurashitov et al., Phys. Rev. Lett. 77, 4708 (1996); Phys. Rev. C 60, 055801 (1999).
[2] SuperKamiokande Collaboration, Y. Fukuda et al., Phys. Rev. Lett. 81, 1562 (1998); 82, 2644 (1999).

[3] LSND Collaboration, C. Athanassopoulos et al., Phys. Rev. Lett. 77, 3082 (1996); 81, 1774 (1998).

[4] S. Weinberg, Phys. Rev. Lett. 43, 1566 (1979).

[5] F. Wilczek and A. Zee, Phys. Rev. Lett. 43, 1571 (1979). 
[6] M. Gell-Mann, P. Ramond, and R. Slansky, in Supergravity, edited by P. van Nieuwenhuizen and D. Z. Freedman (NorthHolland, Amsterdam, 1979); T. Yanagida, in Proceedings of the Workshop on the Unified Theory and the Baryon Number in the Universe, edited by O. Sawada and A. Sugamoto (KEK Report No. 79-18, Tsukuba, Japan,1979); R.N. Mohapatra and G. Senjanovic, Phys. Rev. Lett. 44, 912 (1980).

[7] E. Ma, Phys. Rev. Lett. 81, 1171 (1998).

[8] F. Pisano and V. Pleitez, Phys. Rev. D 46, 410 (1992); P.H. Frampton, Phys. Rev. Lett. 69, 2889 (1992); R. Foot, O.F. Hernandez, F. Pisano, and V. Pleitez, Phys. Rev. D 47, 4158 (1993).

[9] J.C. Montero, C.A.de S. Pires, and V. Pleitez, hep-ph/0103096.

[10] J.C. Montero, C.A.de S. Pires, and V. Pleitez, Phys. Rev. D 64, 096001 (2001).

[11] P.H. Frampton, P.I. Krastev, and J.T. Liu, Mod. Phys. Lett. A 9, 761 (1994).

[12] U. Okamoto and M. Yasue, Phys. Lett. B 466, 267 (1999); T. Kitabayashi and M. Yasue, Phys. Rev. D 63, 095002 (2001); Phys. Lett. B 508, 85 (2001); Nucl. Phys. B609, 61 (2001);
Phys. Rev. D 63, 095006 (2001); J.C. Montero, C.A.de S. Pires, and V. Pleitez, hep-ph/0112203.

[13] See, however, E.J. Chun and S. Pokorski, Phys. Rev. D 62, 053001 (2000); S. Antusch, M. Dress, J. Kersten, M. Lindner, and M. Ratz, Phys. Lett. B 519, 238 (2001), and references therein.

[14] T.P. Cheng and L.-F. Li, Phys. Rev. D 22, 2860 (1980).

[15] G.B. Gelmini and M. Roncadelli, Phys. Lett. 99B, 411 (1981).

[16] R.N. Mohapatra and G. Senjanovic, Phys. Rev. D 23, 165 (1981); C. Wetterich, Nucl. Phys. B187, 343 (1981); E. Ma and U. Sarkar, Phys. Rev. Lett. 80, 5716 (1998).

[17] J.C. Montero, C.A.de S. Pires, and V. Pleitez, Phys. Lett. B 502, 167 (2001); M.B. Tully and G.C. Joshi, Phys. Rev. D 64, 011301 (2001).

[18] R. Foot, H. Lew, X. -G. He, and G. C. Joshi, Z. Phys. C 44, 441 (1989).

[19] J.C. Montero, V. Pleitez, and M.C. Rodriguez, Phys. Rev. D (to be published), hep-ph/0112248.

[20] T.V. Duong and E. Ma, Phys. Lett. B 316, 307 (1993). 